\title{
Development of Statistical Server to Apply Call Center Management Solution
}

IkSoon Kown*, Byung Hyun Choi and Hiesik Kim

* Dept. of Electrical Computer Engineering, University of Seoul Korea

apori@nate.com

\section{Keywords: Statistics, Server, Call Management System}

\begin{abstract}
The study, one of the important solutions in the Call-center about the Call Management System Server and the Call Management System Server than that of the current situation and the advantages and disadvantages of using the measures studied and improved integration of future models of the proposed statistical solution.
\end{abstract}

\section{Introduction}

Call Management System (CMS) is an important solution to provide statistics of the call center system to analyze call volume. This system includes a database, administration, andreporting application to help businesses identify operational issues and take immediate actio to solve them. Using a familiar Windows interface, call center managers can view data and receive customized threshold and exception alerts, all in real time. They can also view historical reports to help them analyze trends, establish performance benchmarks, and plan new marketing or customerservice campaigns. These reports can be easily customized to suit theneeds of the business. With easy access to real-time and historical data, managers can makefaster, better informed decisions, for more effective contact-center operations

The existing systems manufactured by the company's policy are very strict approval process, and accessibility is limited, depending on customer demand for immediate improvement work was inconvenient. To develop their own statistical models to meet the needs of these customers, foreign exchange savings and the company itself in order to maximize the benefits of the model should be developed.

Customer Call Center via the PSTN network if the phone PBX and IVR to answer the phone, enter the information after receiving the customer information to the CTI Server will not send, CTI Server connected to the customer's telephone representatives to consult If the customer information to agent's PC Monitor makes a Display. Recording Server calls between agents and customers start is when the Recording all calls.

Call Management System Server for this event happens in the Call Management System Server Memory When kept in a certain period of time to store the information in the database, and Supervisor Program Manager, using the Call Management System Server and real-time access to monitor data. 


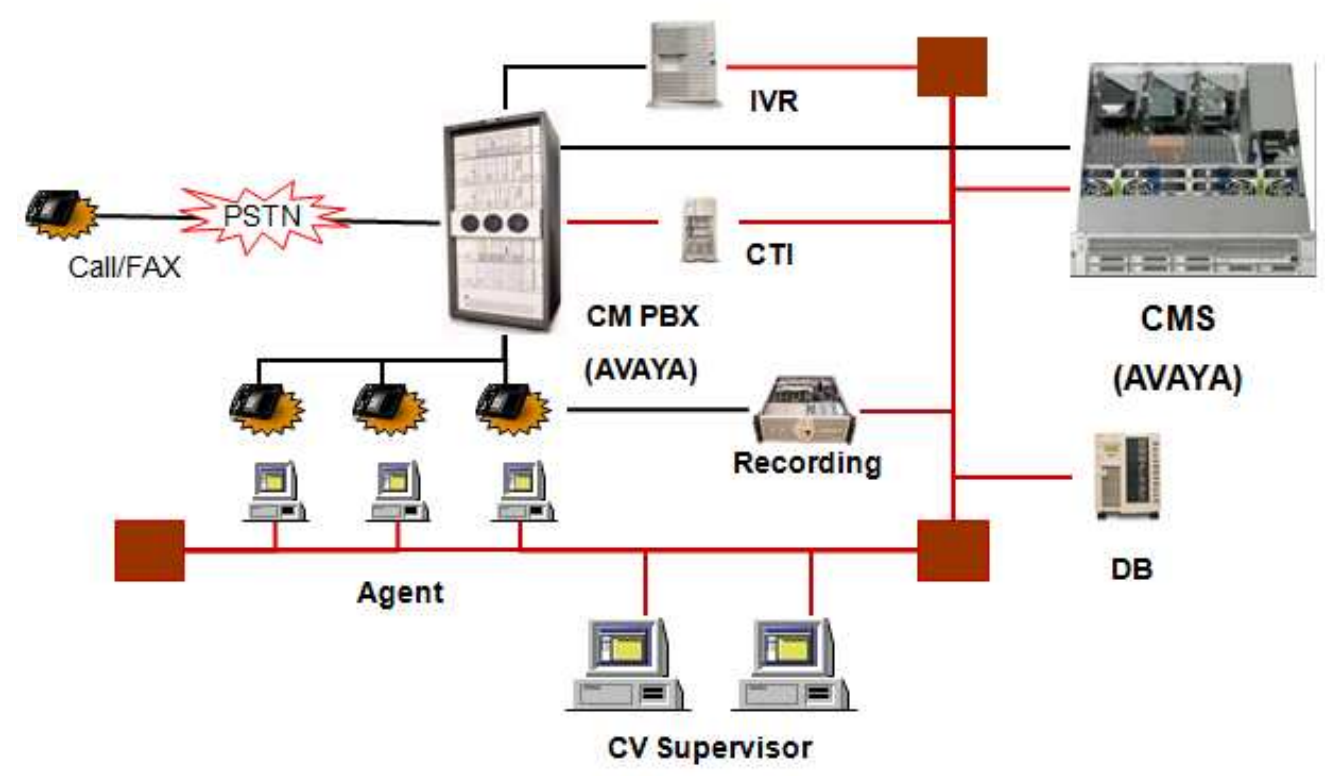

Fig. 1. System frame

\section{CMS Server database configuration}

The Figure 2 below and the Call Management System Server database tables store data for the period as described in the Call Management System Server and IBM's Informix database is installed, largely resides in the memory of the Call Management System Server Real-Time, Real-Time Data at a given time saved as a database based on Historical, Real-Time data and Historical data combined again divided into three ways of Integrated Agent, Skill, VDN, Vector, Trunk / TrunkGroup can be viewed statistics for each of the sections.

Historical Data for Interval, Daily, Weekly, Monthly interval data can be queried in Interval 15 minutes 30 minutes 1 hour Interval jugijung choose one which allows you to specify the period of the Interval specified in the report all the same is applied.

In addition, each storage period, which also fixed, Interval Daily, the maximum 62 days and a maximum value for 5 years (1,825 days) and the Weekly, the maximum 10 years (520 weeks), and if the maximum value of Monthly 10 years (120 months) and save the values ??of the specifications of the Server can be reduced depending on the amount of data.

Call Management System has the software capacity of maximum 8 Telephone exchanger, Average 10000 people agent simultaneously, system 60,000 skill. And it's hardware system is equipped of memory 128GB DRAM, maximum HDD 300GB SAS disk of eight CPU UltraSPARC T2 X 8 (8 core, 64 threads) 


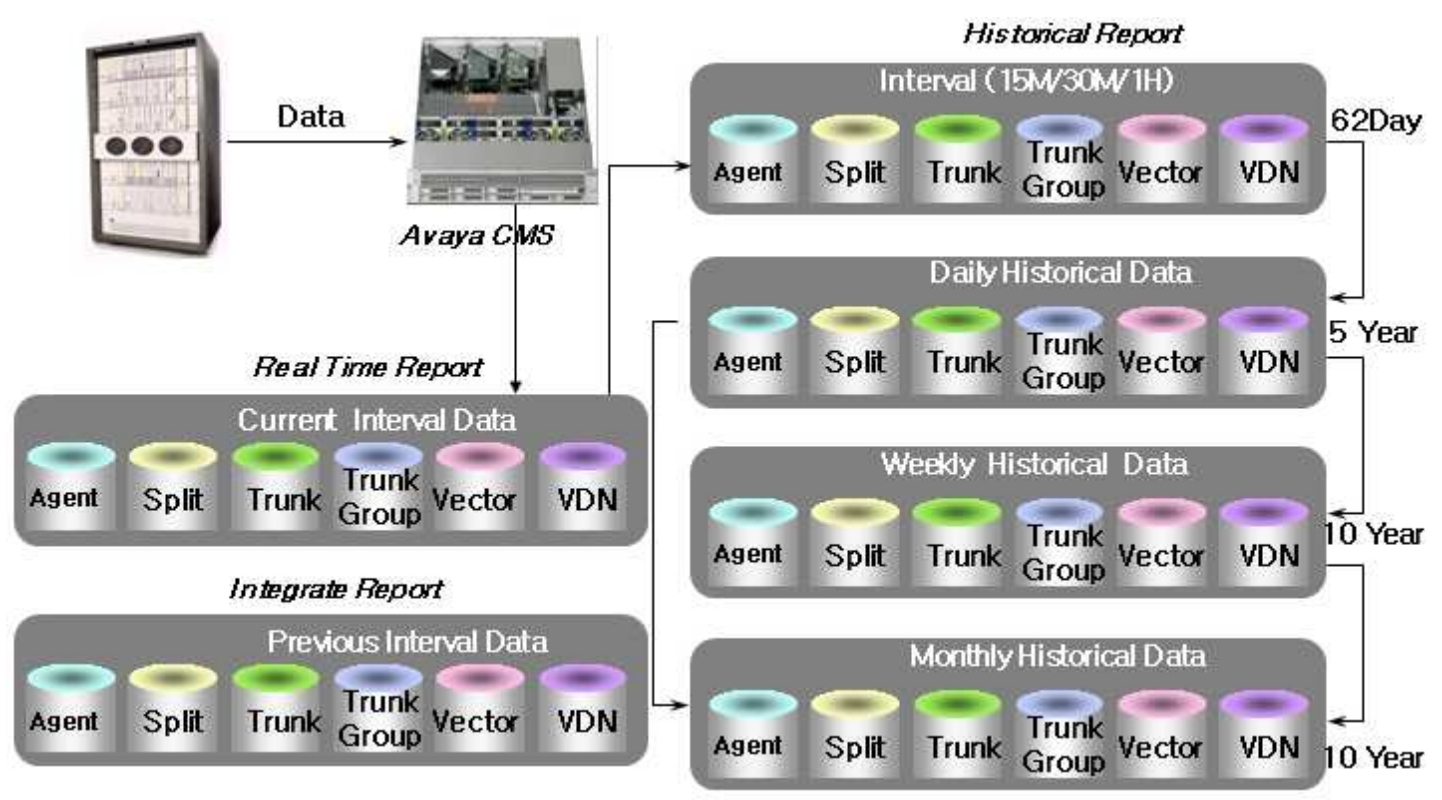

Fig. 2. System server database

\section{Conclusions and Future Research Directions}

This system allows to get immediate feedback, real-time monitoring and customizable alerts to ensure that managers can treat spot problems and respond immediately. The merit is to simplify monitoring and reporting. Because a wizard and other tools make it easy to design customized reports. And managers can schedule the running and printing of reports in comfortable way. So the call center is always being managed easily with the most up-to-date information. This system shows the address of problem areas immediately. If real-time reports show conditions that require immediate changes to keep the contact center operating smoothly, integrated administration enables managers to instantaneously drill into admin to make the required changes.

Real-time call center to operate efficiently, history, and provides integrated data to Call Management System through the GUI Skill / split can be changed easily. Also works with other application via ODBC is easy.

Call Management System Server, but only collects statistics for the Inbound and Outbound call they know nothing about statistics, does not collect data, IVR and CTI nothing about events that happen at has the disadvantage that do not collect statistics.

However, additions or other Agent of the skill and VDN, Trunk, and the ability to monitor the unit's extension number, or CMS Supervisor Who's Online To purchase a license extension shall have the hassle.

These problems after new statistics server built CMS Server's data and CTI, IVR, Outbound receive data by consolidating the existing CMS Server pointed to shortcomings in the new statistics were an improvement on the problem server is the way that you can use to develop. 


\section{References}

[1] Chang-Xing Qi ; Hang Li, Application research of a scalable application gateway model in housing fund call center, International Conference on Computer Design and Applications (ICCDA), 2010, 25-27 June 2010, Volume 5, Page V5-124

[2] Sharma, A.K. ; Juneja, D. ; Bishnoi, C., Intelligent agents in call management system, International Conference on Integration of Knowledge Intensive Multi-Agent Systems, 2005, April 18-21, 2005, Page 9

[3] Sarraf, M., Performance analysis of the Outbound Call Management system, INFOCOM '89. Proceedings of the Eighth Annual Joint Conference of the IEEE Computer and Communications Societies. Technology: Emerging or Converging, IEEE, 23-27 Apr 1989, Page 373

[3] Sarraf, M., Queueing time for the outbound call management system, Global Telecommunications Conference, 1989, and Exhibition. Communications Technology for the 1990s and Beyond. GLOBECOM '89., IEEE, 27-30 Nov 1989, Page 944

[4] Karacali, B. ; Karol, M. ; Krishnan, P. ; Kumar, K. ; Meloche, J., Independent Relays in Overlays for Reliable Enterprise IP Telephony, Sarnoff Symposium, 2006 IEEE, 27-28 March 2006, Page 1

[5] Garg, S. ; Kappes, M., Admission control for VoIP traffic in IEEE 802.11 networks, Global Telecommunications Conference, 2003. GLOBECOM '03. IEEE, 1-5 Dec. 2003, Volume 6 , Page 3514

[6] Wu Chou ; Feng Liu ; Li Li, Web Service for Tele-Communication, International Conference on Telecommunications, 2006. AICT-ICIW '06. International Conference on Internet and Web Applications and Services/Advanced, 19-25 Feb. 2006, Page 88

[7] Anisimov, N. ; Kishinski, K. ; Miloslavski, A., An approach to design distributed CTI applications using Petri nets: an example of a call center, International Conference on Systems, Man, and Cybernetics, 1998. 1998 IEEE, 11-14 Oct 1998, Volume 1, Page 238

[8] Peyravi, F. ; Keshavarzi, A., Agent Based Model for Call Centers Using Knowledge Management, International Conference on Modelling \& Simulation, 2009. AMS '09. Third Asia, 25-29 May 2009, Page 51

[9] Sun Bo ; Zhang Bingyi ; Wang Erzhi ; Sun Liang, Periodic statistical prediction adaptive memory incremental control, International Conference on Industrial Technology, 2008. ICIT 2008. IEEE, 21-24 April 2008, Page 1

[10] Sun-Euy Kim ; Das, C.R., A reliable statistical admission control strategy for interactive video-on-demand servers with interval caching, International Conference on Parallel Processing, 2000. Proceedings. 2000, 2000, Page 135 